\title{
Avances y retos de la economía de la salud
}

\author{
Advances and perspectives in health economics
}

\author{
Patricia Hernández Peña, Armando Arredondo, Claudia Ortiz, Gerald Rosenthal \\ Departamento de Economia de la Salud. Instituto Nacional de Salud Pública - \\ México (P.H.P., A.A., C.O.) \\ Pathfinder International - USA (G. R.)
}

\begin{abstract}
La economía de la salud como especialidad, incorpora la perspectiva económica en el campo de la salud, los servicios, y el complejo médico industrial. Se presenta su evolución desde la perspectiva de sus áreas de interés y su consolidación reflejada en la generación, difusión, réplica y aplicación del conocimiento especializado.
\end{abstract}

Economia de la salud.

\section{Introducción}

En el campo de la salud ha cobrado énfasis la preocupación por asegurar que los recursos se asignen de manera eficiente. Se busca incrementar la producción, y el acceso a los consumidores al menor costo posible y combatir las barreras técnicas, económicas, geográficas, culturales y organizacionales para alcanzar la equidad y la eficiencia de la atencín a la salud.

Esta situación en los países en desarrollo, alcanza mayor urgencia dadas las características demográficas y epidemiológicas, el rezago en la cobertura de servicios y la dinámica de precios internacionales de los insumos para la salud, que agudizan la insuficiencia de los recursos del sector y elevan el costo de los servicios.

La economía de la salud integra los elementos conceptuales y metodológicos que fundamentan e instrumentan la práctica de los principios de eficiencia y equidad en el estudio, y la intervención de la problemática en el complejo médico industrial, los servicios y la salud de la población. La emergencia de esta especialidad, se asocia a una prioridad contemporánea de orden social, técnica y financiera.

Este documento analiza y documenta los avances en el desarrollo y la aplicación de la economía de la salud, a través de sus líneas de trabajo y evidencias de consolidación.

\section{Antecedentes}

Hace 25 años ya se planteaba aprovechar los fundamentos teórico-metodológicos de la economía en la salud. A partir de este esfuerzo, el desarrollo de la especialidad se presentó a través de las innovaciones posteriores en las técnicas y conceptos, asi como en la profundidad y la amplitud de los aspectos en los que se recuperaba la perspectiva economica para el análisis de los problemas de la salud pública.

Los primeros acercamientos se realizaron en la economía laboral, que expresaban el interés de los economistas por explicar la naturaleza de la vida humana, a través de sus ámbitos concretos de trabajo y de la seguridad social, principalmente. Al aplicar las teorías económicas a este campo, se abrieron nuevas maneras de pensar y analizar los servicios de salud. La preocupación fundamental fue desarrollar una estrategia analítica que apoyara el uso óptimo de los recursos. Así se incorporaron los principios de la teoría de la empresa y sus modelos, que facilitaron este proceso.

La necesidad y búsqueda de una óptima asignación de recursos para la salud es un elemento para el cual existe consenso en la sociedad actual. Siempre es mejor el desarrollo de las acciones sin el desperdicio de los recursos, cuya reserva puede aplicarse nuevas acciones. Ahora bien, lo que siempre estará 
en discusión es el criterio de óptimo, ¿por quién se establece? y quién participa en dicha discusion?

Este que es un hecho conocido, va a ser articulado por los economistas. Pero la aplicación del resultado de este análisis hacia la toma de decisiones y la formulación de políticas es más complejo y requiere del conocimiento de otros aspectos. La decisión de quién va a recibir y quién va a sufrir la carencia de los recursos que es planteada explícitamente por el economista siempre se ha presentado en la sociedad. De ahí la preocupación por entiquecer la capacidad de precisar dimensiones que la sociedad no acostumbra expresar. Es inexacto asumir que anteriormente los recursos en salud no eran escasos, 0 que los médicos disponían ilimitadamente de ellos. En realidad se utilizaban libremente para un padecimiento, y al mismo tiempo, no existran en absoluto, eran insuficientes para atender otra enfermedad, o permanecían grupos de población sin cobertura. Esto se mantiene desde los albores de la práctica en salud.

La novedad es el planteamiento explícito de este hecho y la posibilidad de discutirlo, así como la apertura social a reflexionar y presentar argumentos dentro de esta perspectiva. Esto en parte es resultado de una modificación del contexto en un sentido más amplio, pues se ha evolucionado hacia una mayor proximidad hacia los temas económicos, en particular para entender y compartir la preocupación por la asignación optima de los recursos. Cada día los medios masivos van a presentar al público inquietudes y vocabulario que les permiten participar en la discusión. Pero es a partir de esta apertura y la aplicación científica de la Economía de la Salud que se vislumbra la oportunidad de solucionar las inequidades de la atención que hasta hoy se conservan.

El pensamiento economico dentro del ámbito de la salud pública estuvo vinculado en las décadas recientes, a otras ciencias en aspectos particulares como las vertientes de la economía política con el estudio de los determinantes de la salud, la sociología en el análisis de los determinantes del proceso saludenfermedad y la administración, con la evaluación económica y el costeo, que representan la experiencia previa más abundante en la salud pública ${ }^{12}$.

Existe la motivación en este campo, de aprovechar las capacidades analíticas de la economía, y aplicar los modelos económicos para la generación de mayor salud, a comprensión de la dinámica del proceso de salud-enfermedad y mejorar los servicios, a través de la asignación y uso óptimo de los recursos de la salud. Existe para ello un franco compromiso con los objetivos sociales, pues se ha buscado incorporar los valores, y las prioridades del área de la salud a los modelos, lo que impone un reto tanto a la economía como a la ciencias de la salud.

El fundamento inherente con el que se construyen los modelos económicos, el criterio de "ceteris paribus" * es el que define tanto en la salud como en la economía misma, el éxito de la aplicación analítica y la fortaleza de la economía de la salud. De ahí que sea fundamental, utilizar los elementos disponibles, pero sin trivializar los elementos excluídos de los modelos. Esto se vincula a la preocupación por cuantificar, conociendo que lo cuantitativo puede facilitar la discusión, sin detenerse ante las dificultades que imponen los aspectos intangibles, expresados en los esfuerzos por medir la calidad de vida (QUALYS, por ejemplo**). La tendencia a la cuantificación en los análisis, busca eliminar los "ceteris paribus" de manera creciente.

Si se considera que existe una relación inversa en cuanto al poder analítico y la integralidad de los métodos, es posible aceptar que el análisis económico es limitado pero poderoso. De ahí la importancia de aceptar las limitaciones y aplicar de manera flexible y lo más real posible, las conclusiones. La economía de la salud por lo tanto, acepta cuantificar, para manejar ciertas incertidumbres.

La emergencia de una especialización en economía de la salud, se justifica porque si bien el propósito en su forma más simple es aplicar los modelos económicos a este campo, no busca realizar un análisis económico con los datos de salud, sino incorporar los valores de las instituciones, de la sociedad y analizar e intervenir en el contexto real, donde actúa el mercado. Esto es, el reto que se asume es incorporar dimensiones, tales como el análisis e intervención en las externalidades*** de la salud. Si se considera algún otro bien, el consumidor no requiere de tanto apoyo, como en salud, donde la ignorancia relativa de los consumidores es un factor que justifica un tratamiento económico diferente.

La especialidad ha sido básica para romper algunos supuestos falso que predominaban en los servicios de salud. Este es el caso de la demanda de atención, donde se asumía que al producirse los servicios, serían consumidos. Con frecuencia, la modificación del horario de atención, según las

\footnotetext{
* "Ceteris Paribus": Las demás cosas constantes. Se utiliza en economía para controlar los aspectos que no se conocen o se asume que no se modifican en el análisis. El escrito hace referencia al desconocimiento de aspectos importantes, pero que por insuficiencia de informacion, deben permanecer fuera de los modelos de análisis (Samuelson, $P^{16}, p 9$ ).

** QUALYS (Quality Adjusted Life Years): Unidad que ajusta los años de vida ganados por una intervención en la calidad de vida del individuo. Es decir, mide los beneficios intangibles como reducir el dolor, las consecuencias de una enfermedad en el bienestar del individuo, entre otros. La técnica que generalmente se utiliza para moneterizar este tipo de condiciones es la disponibilidad para pagar (Gudex ${ }^{9}$ ).

*** Se entiende por externalidad, a los efectos de la actividad económica en terceras personas, que no participan en el intercambio.
} 
necesidades y la disponibilidad de tiempo de los usuarios, aseguran una mayor utilización de los servicios. La articulacion demanda-oferta de atención representa una área de trabajo particular que se vincula con la comprensión de que su producción es materia pública.

La búsqueda de la contención de costos por ejemplo, es muy útil porque permitirá liberar recursos que actualmente pueden estar siendo desperdiciados. Sin embargo esta condición económica no es el objetivo del sistema. El proposito es que la gente reciba atención cuando la necesita, y no se utilicen recursos innecesariamente. Este es el caso de la elevación en el número de días de estancia hospitalaria, como resultante de la inadecuada planeación en los servicios de apoyo de radiología o laboratorio.

El campo de la salud manifiesta permanentemente sus particularidades inherentes, que han servido de estímulo a un desarrollo especializado. Por ejemplo, cuando se analiza el uso de recursos, se enfrenta a la dificultad en la identificación de los productos. $¿$ Cómo medir el producto de los servicios múltiples? Las complejidades del análisis han sido resueltas tanto teórica como instrumentalmente. Este avance la llevó a su consolidación como especialidad.

\section{Consolldaclón de la Especialidad}

La base conceptual de todas las disciplinas científicas, evoluciona permanentemente. Esta dinámica se agiliza en la interacción con las otras áreas del saber, con la presencia de nuevas necesidades de aplicación y cuando existen recursos humanos que asumen el reto de buscar crecientes niveles de comprensión, explicación y transformación de la reali$\mathrm{dad}^{8}$. Puede decirse que una especialidad se ha consolidado cuando existe un cuerpo de conocimiento que es replicado y difundido por una masa crítica de cientíticos que lo maneja. Actualmente esta especialidad se considera consolidada debido a su presencia en el quehacer científico y sus logros operacionales en los servicios de salud:

\section{Presencla de un Cuerpo de Conocimlento}

La integración de un cuerpo sólido y reconocido de elementos teóricos y metodológicos brinda el primer respaldo y el punto de partida en la constitución de esta especialidad. Se busca responder a las dificultades inherentes al campo, vinculadas al hecho de que la salud es una área en la cual existen planteamientos técnicos, sociales, políticos, culturales y éticos, diferentes de otras esferas de la producción y el mercado de bienes y servicios.

Los ámbitos de intervencíón de la teoría y la metodología económica en la salud pública incluyen el nivel microeconómico de la empresa, aprovechan los elementos involucrados en las dinámicas del mercado, y operan en el nivel político, eminentemente macroeconómico y social, lo que brinda la posibilidad de una mayor participatión en la toma de decisiones de índole sectorial y extrasectorial, esto es, no sólo en los servicios de salud, sino también de sus determinantes, y el complejo médico industrial, que incluye principalmente al mercado de insumos, medicamentos y tecnología. El cuerpo teórico y metodologico de la especialidad se ampliará en la medida en que la aplicación de los principios de la economía en el campo de la salud sea experimentada con sentido crítico y evaluativo.

Existe una propuesta de sistematización de las áreas de trabajo potenciales, que si bien no es la única modalidad de agrupamiento factible, facilita la comprensión de la materia mediante la integración de nueve grandes áreas que van desde los determinantes del proceso salud-enfermedad; la salud como un bien económico; la demanda; la oferta de sevicios; el equilibrio de mercado; los instrumentos de política económica; la evaluación microeconómica; la evaluación general del sistema; y su aplicación en los procesos de planeación y organización de los servicios ${ }^{13}$.

\section{Producción y Dlfusión del Conocimiento}

El cuerpo teórico de la economía de la salud expandió su aplicación principalmente durante la década de los ochenta. Durante esa década el cuerpo de conocimientos de la economía de la salud, asume una presencia propia, que da pie a la innovacion, la adaptación y práctica, que genera mayor preocupación por la apropiación e intercambio del marco de referencia teórico y metodologico existente. Un reflejo de este proceso es el crecimiento en las publicaciones especializadas.

A nivel internacional la primera revista especializada en economía de la salud empez 6 a publicarse en $1982^{14}$. La producción científica sobre el tema, ha sido relativamente escasa en países de América Latina y el Caribe, a diferencia de lo que ocurre en Europa, Canadá y Estados Unidos de Norteamérica, donde el auge de publicaciones en la materia se había iniciado con anterioridad. Esto además es consistente con lo que ocurre en otras especialidades de las ciencias sociales.

La producción contemporánea de publicaciones en esta área ha mostrado un crecimiento progresivo. Dentro del Current Contents ${ }^{5}$ existen 37 publicaciones cuyo nombre se asocia con la economía de la salud y 147 son relacionables.

Se realizó una búsqueda bibliográfica utilizando el nombre de "Economía de la Salud" para ingresar 
tanto en la base de datos LILACS como en MEDLINE. El período analizado comprende de 1982 a 1993 en la primera base y de 1982 a mayo de 1995 en la segunda. De ahí se obtuvo un listado de 12.128 referencias en total; 12.075 corresponden a MEDLINE y 53 a LILACS (Tabela 1). Posteriormente se solicitaron listados de la producción bibliográfica para las principales áreas temáticas de la especialidad.

La ubicación de los artículos no siempre es exclusiva a un área en un sentido estricto, por lo que se eligió sólo una de ellas a partir del título. Por ejemplo, para el año de 1992 están registrados en MEDLINE como análisis de costos, un total de 508 artículos, pero muchos de éstos se encuentran clasificados según su área sustativa. Este procedimiento

Tabla 1- Artículos sobre economía de la saiud en MEDLINE y LILACS.

\begin{tabular}{lcccc}
\hline $\begin{array}{l}\text { Fuente de } \\
\text { Información }\end{array}$ & $1982-1992$ & 1993 & 1994 & 1995 \\
\hline LILACS' $^{*}$ & 42 & 11 & - & - \\
MEDLINE & 8.976 & 1.550 & 1.020 & 529 \\
\hline Total & 9.018 & 1.561 & 1.020 & 529 \\
\hline
\end{tabular}

Fuente: U.S.National Library of Medicine (MEDLINE). Diciembre 1992, 1993, 1994 y enero-mayo 1995. Centro Latinoamericano y del Caribe en Ciencias de la Salud (LILACS) 1982-1992 y 1993.

"La base de datos de LILACS no dispone de información para los años de 1994 y 1995.

los subclasifico en particular, por costo-beneficio, costo-efectividad, costo-utilidad*, etc. Por lo que respecta a LILACS, sólo se tienen 6 estudios de costos en el período.

El número de artículos explícitamente dedicado a la economía de la salud se ha incrementado aceleradamente hasta duplicarse en un período de diez años, marcando una tendencia de expansión. Existe un mayor número de artículos sobre el tema dentro de la base de datos de MEDLINE en comparación con LILACS, fenómeno común en otras disciplinas.

Al analizar la información registrada en las bases de datos de las publicaciones se evidencia un predominio de temática por año, siendo para 1993 las publicaciones más frecuentes, las de evaluación económica, en sus diversas vertientes; financiamiento; planeación; y organización (Tabela 2).

Resulta evidente también, la disparidad en el

* Todas estas formas de evaluación económica buscan explicitar comparativamente los costos y los beneficios en la asignación altermativa de recursos. Las formas de evaluar los costos son monetarizadas, pero los beneficios podrán adoptar modalidades diversas: monetarias, de comportamiento, o de salud. desarrollo de la especialidad entre los países. Si se analizan a través de la clasificación de Banco Mundial según nivel de ingreso en 1993, se observa que las publicaciones en ambas bases de datos, proceden, el $96 \%$ de los artículos de los países reportados con ingreso alto (1.507 artículos, de 20 países), $2 \%$ con ingreso medio alto ( 32 artículos de 6 países), $0,5 \%$ de los países con ingreso medio bajo ( 8 artículos de 5 países), $0,6 \%$ de bajo ingreso ( 9 artículos de 4 países) (Tabela 3).

\section{Reproducclón del Conocimlento}

En lo referente a los programas docentes, su expansión se encuentra en proceso. Desde la década de los cincuenta existían economistas que trabajaban en salud, y personal de salud que aplicaba criterios económicos, pero la presencia de un cuerpo de conocimientos validado y de personal especializado "Ad Hoc" data de la década de los setenta. La docencia y formación de recursos humanos con programas académicos surgió en Europa y Estados Unidos de Norteamérica, donde se han capacitado recursos de otras regiones que laboran en diferentes instituciones y países. Actualmente la profesionalización de la especialidad se basa en los programas de postgrado a nivel de especialidad, maestría, doctorado y educación contínua.

La formación de recursos humanos especializados ha sido considerado un proceso prioritario ${ }^{7,15}$. Los contenidos de la economía de la salud se desarrollan en prácticamente todos los programas de formación de recursos en salud pública, además de existir terminales específicas.

\section{Presencla de una Masa Crítica}

Existen grupos de trabajo en todos los continentes. Son ejemplo de ello en Europa, los grupos del Centro de Investigación en Economía de la Salud, CREDES en Francia ${ }^{4}$, la Organización de Cooperacion y Desarrollo, OCED, la Unión Europea, Portugal, España, y la escuela inglesa con los grupos de York ${ }^{3}$, Birminghan y Londres, que tienen una larga tradición en su estudio. En América, Canadá en Montreal y Hamilton ${ }^{2,12}$, y los Estados Unidos, en las universidades de Boston, Hopkins,Seattle, George Washington, entre otras.

Dentro de América Latina existen grupos de trabajo en la especialidad, con avances diferentes, en: Brasil con grupos en Rio de Janeiro, Sao Paolo y Brasilia, República Dominicana, Haití, Uruguay, Chile, Argentina, Venezuela, Panamá, Costa Rica, Cuba y México.

El catálogo de economistas de la salud en intercambio, para 1993 mostró registrados un número 
Tabla 2 - Artículos de acuerdo al ámbito de aplicación de economía de la salud.

\begin{tabular}{|c|c|c|c|c|c|c|}
\hline \multirow[t]{2}{*}{ Ámbito de Aplicación } & \multicolumn{3}{|c|}{ MEDLINE } & & \multicolumn{2}{|c|}{ LILACS } \\
\hline & 1992 & 1993 & 1994 & 1995 & 1992 & 1993 \\
\hline $\begin{array}{l}\text { Determinantes de la Salud } \\
\text { Demanda de Servicios } \\
\text { Oferta de Servicios } \\
\text { Evaluación Económica }\end{array}$ & $\begin{array}{l}30 \\
77 \\
43\end{array}$ & $\begin{array}{c}9 \\
96 \\
41\end{array}$ & $\begin{array}{l}5 \\
71 \\
39\end{array}$ & $\begin{array}{l}2 \\
37 \\
13\end{array}$ & $\begin{array}{l}2 \\
1\end{array}$ & 1 \\
\hline $\begin{array}{l}\text {-Costo-beneticio } \\
\text {-Costo-efectividad } \\
\text {-Costo-utilidad } \\
\text {-Tecnologia } \\
\text {-Calidad } \\
\text { Política Económica }\end{array}$ & $\begin{array}{c}4 \\
35 \\
2 \\
175 \\
196\end{array}$ & $\begin{array}{c}1 \\
56 \\
721 \\
55 \\
284\end{array}$ & $\begin{array}{c}142 \\
40 \\
2 \\
52 \\
185\end{array}$ & $\begin{array}{c}69 \\
36 \\
1 \\
24 \\
104\end{array}$ & $\begin{array}{l}1 \\
6\end{array}$ & 2 \\
\hline $\begin{array}{l}\text {-Instrumentos de Política } \\
\text {-Financiamiento } \\
\text {-Equidad }\end{array}$ & $\begin{array}{c}420 \\
163 \\
15\end{array}$ & $\begin{array}{c}39 \\
197 \\
18\end{array}$ & $\begin{array}{c}117 \\
169 \\
15\end{array}$ & $\begin{array}{l}19 \\
59 \\
7\end{array}$ & $\begin{array}{c}19 \\
9\end{array}$ & $\begin{array}{l}1 \\
5 \\
1\end{array}$ \\
\hline $\begin{array}{l}\text { Planeación y Organización } \\
\text { Informes }\end{array}$ & 4 & $\begin{array}{l}94 \\
17\end{array}$ & $\begin{array}{c}171 \\
12\end{array}$ & $\begin{array}{c}149 \\
9\end{array}$ & $\begin{array}{l}1 \\
3\end{array}$ & 1 \\
\hline Total & 1.244 & 1.550 & 1.020 & 529 & 42 & 11 \\
\hline
\end{tabular}

Fuente: U.S. National Library of Medicine (MEDLINE). Diciembre 1992, 1993, 1994 y mayo-junio 1995. Centro Latinoamericano y del Caribe en Ciencias de la Salud (LILACS) 1982-1992 y 1993.

aproximado de 188 especialistas. De ellos, 90 proceden de países del primer mundo, aún cuando un subgrupo desarrolla actividades en otros países; 53 corresponden a Asia; 34 son de Africa; y 11 de países de América Latina y el Caribe ${ }^{10 .}$

\section{Aplicación del Conocimlento}

La problemática analizada en diferentes ámbitos del mundo, en particular en los países de alto ingreso, puede ser similar a la de otros países, pero amerita de tratamiento diferente, además de presentar prioridades y particularidades que no coinciden necesariamente. El cuerpo térico-metodológico de la Economía de la Salud puede brindar respuesta a las interrogantes y problemas específicos de cada región. Sin embargo, el aprovechamiento de los hallazgos y metodologías ameritan de un esfuerzo de adaptación, adecuación y desarrollo local, a fin de elevar el éxito en su aplicación en el corto plazo.

La aplicación del conocimiento en las políticas de salud tambien se ha incrementado, a traves de las modificaciones en el financiamiento de los sistemas de salud y la operación de los instrumentos de política económica prácticamente a nivel mundial: El análisis del papel del Estado en la prestación de servicios de salud, logra una aplicación amplia de la especialidad, definiendo prioridades y operando los instrumentos de política económica en la regulación ambiental, las modalidades de provisión directa de los servicios, el financiamiento y la selectividad de programas prioritarios, entre otros, como producto de las reformas dentro de este campo en muchos países ${ }^{6}$. Una de las principales estrategias es aprovechar la potencialidad de los servicios de salud del sector privado y asegurar su operación coordinada con la atención pública y la seguridad social ${ }^{1}$.

Existen esfuerzos importantes para operacionalizar la llamada mezcla pública-privada de financiamiento en América Latina, a partir del pago por servicios, diferenciado por niveles de ingreso, atendiendo a su relevancia como fuente de recursos, y combinando formas de prestación de servicios e infraestructura financiadas en forma pública o privada. Para ello la economía de la salud está en el proceso de depurar y evaluar los criterios operativos de asignación de recursos.

La comunicación, intercambio e incidencia en la toma de decisiones estratégicas en salud, puede ampliarse aún más, potenciando sus logros. Para ello en diferentes países se ha iniciado la constitución de asociaciones nacionales de economía de la salud, como la brasileña, la española y la portuguesa, que impulsan su análisis y aplicación.

\section{Conclusiones}

La evolución de la economía de la salud muestra su presencia como una especialidad que se ha consolidado, pero que aún enfrenta grandes retos. El primero es tal vez que sus avances y logros no son homogéneos en el mundo, existiendo mayor desarollo en los países más avanzados, al igual que en otras especialidades.

Sin duda se presentan una serie de dificultades para la generación y reproducción del conocimiento, así como para la utilización de resultados de investigación donde el análisis económico sea el hilo conductor, tanto en organizaciones gubernamentales 
Tabla 3 - Articulos según país de origen de las publicaciones y por nivel de ingreso.

Nivel de ingreso/pais

MEDLINE

\begin{tabular}{ccc} 
& \multicolumn{2}{c}{ MEDLINE } \\
\hline 1992 & 1993 & 1 \\
& & \\
803 & 1.020 & 7 \\
171 & 229 & 135 \\
40 & 33 & \\
28 & 45 & \\
17 & 27 & \\
16 & 23 & \\
15 & 9 & \\
14 & 17 & \\
8 & 3 & \\
8 & 30 & \\
7 & 10 & \\
7 & 10 & \\
3 & & \\
5 & 5 & \\
21 & 10 & \\
12 & 12 & \\
1 & 1 & \\
1 & 1 & \\
1 & 7 & \\
1 & 5 & \\
& 3 &
\end{tabular}

Ingreso Alto

Estados Unidos

Inglaterra

Suecia

Canadá

Suiza

Australia

Dinamarca

Noruega

Italia

Alemanha

Francia

España

Hong-Kong

Japón

Nueva Zelandia

Países Bajos

Irlanda

Singapur

Israel

Finlandia

Austria

$1994 \quad 1995$

LILACS

Ingreso Medio-Alto

Brasil

Hungria

Sudáfrica

Puerto Rico

Argentina

México

Rusia

Bélgica

Portugal

$746 \quad 337$

$135 \quad 74$

$15 \quad 13$

$24 \quad 12$

$10 \quad 10$

$9 \quad 7$

45

103

$3 \quad 3$

$19 \quad 14$

61

62

1

29

$7 \quad 3$

$9 \quad 17$

1

$1992 \quad 1993$

$\begin{array}{lll}77 & 7\end{array}$

Corea

Ingreso Medio Bajo

Panamá

Chile

Polonia

Nuova Guinea

Rumania

Cuba

Tailandia

Jamaica

Ucrania

Checoslovaquia

Ingreso Bajo

Kenia

Nicaragua

India

Egipto

Pakistán

Nigeria

Zimbabwe

\begin{tabular}{|c|c|}
\hline $\begin{array}{l}3 \\
7 \\
2\end{array}$ & $\begin{array}{c}3 \\
13\end{array}$ \\
\hline $\begin{array}{c}6 \\
31\end{array}$ & $\begin{array}{l}5 \\
8 \\
1\end{array}$ \\
\hline
\end{tabular}

1

8

2

7

129

$\begin{array}{ll}3 & \\ 2 & 4\end{array}$

2

2

1

1

3

2

Total

1.244

2

1

2

7

1

1

2

1

1

1

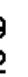

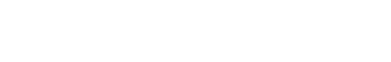

Corresponde a la información de enero-mayo de 1995.

Fuente: U. S. National Library of Medicine (MEDLINE). Diciembre 1992, 1993, 1994 y enero-mayo de 1995.

Centro Latinoamericano y del Caribe en Ciencias de la Salud (LILACS) 1992-1993 World Bank Social Indicator of development, U.S.A., 1993. 
como no gubernamentales y principalmente en pafses en desarrollo. Esto conlleva problemas de muy diverso tipo, que pueden ser financieros y de escasez de recursos, donde las posibilidades y estrategias de la especialidad en la capacidad de canalizar y asignar eficientemente recursos, son puestas a prueba para proseguir su propio desarrollo.

En otra vertiente figura el compromiso con lo social y los valores inherentes a la salud, que incluyen todo un espectro de factores culturales y políticos, no sólo técnicos, que deben ser incorporados con mayor eficiencia en los análisis económicos. Por otra parte, la evolución de estos factores y su complejidad, representan el mecanismo básico por el que esta especialidad identifique sus prioridades de investigación e intervención en cada sociedad.

Es justamente dentro de este contexto de dificultades de generación, difusión y aplicación del conocimiento especializado, que visualizamos los mayores retos que se presentan ante la economía de la salud. Se hace necesario que se impulse su desarrollo con sentido evolutivo para lograr que llegue a ser una herramienta técniça de análisis, cuyo amplio uso brinde alternativas de solución a los problemas de salud pública en su campo, particularmente en lo referente a la eficiencia, la equidad y la calidad de los servicios de salud que se producen.

Hacer de la economía de la salud una herramienta de análisis relevante en la organización de los servicios de salud, y su interacción con el entorno, con la respuesta social organizada y las condiciones de salud del conjunto de la población, amerita de un avance simultáneo en cuanto a la generación, reproducción y aplicación de su cuerpo teórico-metodológico. Se requiere de promover la investigación en el campo y la utilización de sus resultados de investigación, que sean difundidos en programas docentes, y asegurar su contribución crítica creciente al análisis de la problemática económica en el sector. La progresiva resolución técnica ampliará su campo de aplicación hacia nuevas áreas de intervención.

\section{Referenclas Bibliográficas}

1. BENNET, S. The mystique of markets: public and private health care in developing countries. London (Public Health and Policy Dep. Publ. 4), 1991.

2. CENTRE OF HEALTH ECONOMICS AND POLICY ANALYSIS - Annual Report 1989-1990. Hamilton, Ontario, 1991.

3. CENTRE FOR HEALTH ECONOMICS University of New York. Health economics consortium. New York, 1991. (Discussion paper 80).

4. CENTRE DU RECHERCHE ET ÉTUDES DU ECONOMIE DE LA SANTÉ - Socioeconomie de la santé 1990-3. Paris, 1991.

5. CURRENT CONTENTS: Behavioral and Social Sciences. Philadelphia, ISI, 1992.

6. DEPARTMENT OF HEALTH AND HUMAN SERVICES DHHS. Healthy people 2000. Washington, 1990.

7. FERREIRA, J. El liderazgo en salud pública en América. Educ. méd. Salud, 23(1): 5-13, 1989.

8. FRENK, J. The new public health. Annu. Rev. Publ. Health, 14: 469-90. 1993.

9. GUDEX, C. Qualys and their use by the health services. New York, Centre for Health Economics of University of York, 1986.(Discussion paper $\mathrm{n}^{\circ} 20$ ).

10. HEALTH economics exchanges 1993 directory. Londres, Universidad de Londres, 1993.

11. McMASTER UNIVERSITY. Report on economics research., Hamilton, Ontorio, 1989. (Working papers series 89-100)

12, MEDICI, A. C. Las contribuciones de la economfa de la salud a la investigación en salud. In: Ciencias sociales y salud en América Latina. Montevideo, Ed. NUNES/OPSCIESU, 1986. p.183-96.

13. MILLS, A. \& GILSON, L. Health economics for developing countries: a survival Kit. Oxford. 1988. (EPC Publication $n^{\circ} 17$ ).

14. MUSGROVE, P. Economia de la salud: perspectivas para América Latina. Washington, D. C. 1989. (OPS - Publicación Cientifica $\left.\pi^{\circ} 517\right)$.

15. ORGANIZACIÓNPANAMERICANADE LASALUD.Orientaciones estratégicas y prioridades programáticas para la OPS en el cuadrienio 1991-1994. Washington D. C., 1990.

16. SAMUELSON, P. N, Economfa. 12' ed. Mexico, Mac GrawHill, 1986.

\section{Abstract}

Health economics is a specialized field of economic science that applies the economic perspective to the fieids of health, the medical-industrial complex and health services. A brief review of the evolution of this speciality by subject, as well as the level achleved assessed in terms of generation, diffusion, reproduction and application of its specialized knowledge, is presented.

Health economics.

\section{Resumo}

A economia da saúde incorpora a p8erspectiva económica no campo da saúde, os serviços e o complexo médico-industrial. Foi apresentada a evolução desse campo, desde a perspectiva das áreas de interesse es avanços para sua consolidaçajo, partindo dos seguintes aspectos: generaçă difusăo, réplica e aplicaçăo do conhecimento especializado.

Economia da saúde. 